\title{
Relapse rates of inflammatory bowel disease patients in deep and clinical remission after discontinuing anti-tumor necrosis factor alpha therapy
}

\author{
Hlavaty T, Krajcovicova A, Letkovsky J, Sturdik I, Koller T, Toth J, Huorka M \\ 5th Department of Internal Medicine, Sub-department of Gastroenterology and Hepatology, Faculty of Medicine \\ Comenius University Bratislava and University Hospital Bratislava, Slovakia. tibor.hlavaty2@gmail.com
}

\begin{abstract}
BACKGROUND AND AIMS: Relapse rates after discontinuing anti-tumor necrosis factor- $\alpha$ (TNF $\alpha$ ) therapy of inflammatory bowel disease (IBD) patients in deep remission are poorly understood. This prospective singlecenter open-label study evaluated the relapse rates of IBD patients after stopping anti-TNFa therapy.

METHODS: All IBD patients who were in clinical remission and stopped anti-TNFa therapy in 2011-2013 and were followed up for at least 12 months were enrolled. The "Ultradeep" patients were in calprotectin-negative $(<50 \mathrm{ng} / \mathrm{g}$ ) deep remission for at least six months and ceased anti-TNFa therapy on physician recommendations. The "clinical" patients were in clinical but not deep remission and ceased anti-TNFa therapy for other reasons. Relapse rates were assessed and relapse risk factors identified.

RESULTS: One year after stopping, $27 \%$ and $27 \%$ of the Ultradeep $(n=11)$ and Clinical $(n=11)$ patients relapsed, respectively. Two years after stopping, $57 \%$ and $62 \%$ relapsed, respectively $(p=0.89)$. All relapsed patients who underwent retreatment with anti-TNFa therapy re-entered remission. Male sex was a significant risk factor for relapse $(p=0.03)$.

CONCLUSION: Our study showed that even highly selected IBD patients who lack clinical, endoscopic or laboratory signs of disease activity have a relatively high relapse rate in the follow-up period after ceasing anti-TNF $\alpha$ therapy (Tab. 2, Fig. 3, Ref. 24). Text in PDF www.elis.sk.

KEY WORDS: Crohn's disease, ulcerative colitis, infliximab, adalimumab, treatment discontinuation, relapse rate.
\end{abstract}

\section{Introduction}

Inflammatory bowel diseases (IBD) include ulcerative colitis (UC) and Crohn's disease (CD), and are idiopathic chronic relapsing inflammatory conditions of the gastrointestinal tract. Biological therapy with monoclonal antibodies against tumor necrosis factor $\alpha($ anti-TNF $\alpha)$ such as infliximab and adalimumab have significantly improved the management of CD and UC patients (1-5). However, anti-TNF $\alpha$ therapy has some risks and burdens. Firstly, since it is essentially a specific immunosuppressive therapy, there is ongoing concern that it could aggravate latent or concomitant infections and/or compromise oncological immune surveillance. Indeed, although anti-TNF $\alpha$ monotherapy is considered safe, the risk of infections, especially opportunistic infections, is increased when it is given in combination with other immunosuppressives

5th Department of Internal Medicine, Sub-department of Gastroenterology and Hepatology, Faculty of Medicine Comenius University Bratislava and University Hospital Bratislava, Slovakia

Address for correspondence: T. Hlavaty, MD, PhD, IBD Centre, Department of Internal Medicine V, Sub-department of Gastroenterology and Hepatology, University Hospital Bratislava, Comenius University Bratislava, Ruzinovska 6, SK-826 06 Bratislava, Slovakia.

Phone/Fax: +421.2.48234905

Acknowledgements: This work was supported by the University hospital Bratislava (BA092011). or corticosteroids (6-8). Secondly, over time, there is a cumulative increase in secondary loss of response, largely because of the development of blocking anti-drug antibodies. Last but not least, therapy with anti-TNF $\alpha$ remains an expensive treatment (9).

Given the potential risks and burdens of long-term biological therapy, it has been asked how long it should be continued and when it can be safely stopped (10). However, the data on these issues are scarce and conflicting, and consequently generally accepted stopping rules have not yet been developed. At the time when the current study was initiated, there were only a few observational clinical studies that examined the relapse rates of $\mathrm{CD}$ patients after infliximab withdrawal. One was a prospective observational study with 46 patients from Domenech. The cumulative relapse rate at 1 year in the latter study was reported to be $43 \%$ (11). Similarly, the study of 48 patients by Waugh et al. found cumulative relapse rates at 1 and 2 years of $42 \%$ and $65 \%$, respectively (12). The prospective multicenter open-label STORI trial with 110 wellcharacterized CD patients who stopped infliximab while continuing with azathioprine reported cumulative relapse rates of $43.9 \%$ and $52.2 \%$, respectively within the first and second years (13). Multivariable analysis showed that risk factors for relapse included male sex, absence of surgical resection, leukocyte counts $>6.0 \times$ $10^{9} / \mathrm{L}$, hemoglobin levels $\leq 145 \mathrm{~g} / \mathrm{L}, \mathrm{C}$-reactive protein levels $\geq$ $5.0 \mathrm{mg} / \mathrm{L}$, and fecal calprotectin (FC) levels $\geq 300 \mu \mathrm{g} / \mathrm{g}$. FC levels were in particular the most significant predictor of relapse in 
the univariate analyses which showed that 15 of 21 patients ( $71 \%$ ) whose baseline FC levels were $\geq 300 \mu \mathrm{g} / \mathrm{g}$ relapsed within 2 years compared to $25 / 64$ (39\%) of patients with FC levels $<300 \mu \mathrm{g} / \mathrm{g}$ $(\mathrm{p}=0.0002)$ (13). Calprotectin is a cytoplasmic protein in neutrophils and its serum concentrations correlate with the migration of these cells to the intestinal lumen REF Calpro. It is thus a very sensitive marker of intestinal inflammation.

The paucity of data on the disease course in IBD patients after anti-TNF $\alpha$ therapy discontinuation has led us to conduct a prospective clinical study on IBD patients in clinical remission. The primary aim of this study was to evaluate the cumulative relapse rate within 1 year after stopping anti-TNF $\alpha$ therapy in low-risk IBD patients who were in corticosteroid-free deep remission with endoscopic mucosal healing and normal FC levels $(<50 \mathrm{ng} / \mathrm{g})$. The second study aim was to compare the relapse rates of this group with the relapse rates of a second group of IBD patients who were in clinical but not deep remission at the time they stopped anti-TNF $\alpha$ therapy.

\section{Methods}

\section{Study design and cohorts}

This prospective single-center open-label trial was conducted at the tertiary IBD center in a university hospital in Bratislava, Slovakia, between October 2011 and November 2013. The study evaluated the relapse rate after anti-TNF $\alpha$ therapy cessation in two groups of consecutive IBD patients who had attained clinical remission after at least 12 months of biological therapy. The Ultradeep group formed the prospective interventional study arm and consisted of IBD patients in FC-negative deep remission who fulfilled the eligibility criteria described below and who deliberately ceased their anti-TNF $\alpha$ therapy after consenting to participation in the study. The Clinical group formed an observational study arm and consisted of IBD patients who did not fulfill the eligibility criteria of the Ultradeep cohort but nevertheless stopped anti$\mathrm{TNF} \alpha$ therapy for other reasons, and at the time of cessation were in clinical but not deep remission. All patients were followed up for at least 12 months. The demographic and clinical characteristics (age, disease duration, disease location, disease behavior, as defined by the Montreal classification, concomitant medications, and previous IBD-related surgeries) were extracted (14).

\section{The Ultradeep cohort}

These patients had either CD or UC and fulfilled all inclusion criteria: they were over 18 years of age, they had been treated with biological therapy (either infliximab or adalimumab) for at least 12 months, they were in deep remission at the time of enrollment (defined below), they had negative FC levels (defined as $<50$ $\mathrm{ng} / \mathrm{g}$ of stool) at the time of enrollment, and they had consented to ceasing biological therapy and participating in the study after an in-depth discussion of the risks and benefits with their physician. In addition, if the patients had $\mathrm{CD}$, they could not have an active fistula at the time of enrollment: they had to either lack the history of fistula or else it had to be healed with no discharge and no active opening. Deep remission was defined as corticosteroid-free clinical remission for 6 months before enrollment with mucosal healing on endoscopy at the time of inclusion, no signs of activity on abdominal ultrasound, computed tomography enterography, or magnetic resonance imaging enterography, and with negative $\mathrm{C}$ reactive protein levels $(<5 \mathrm{mg} / \mathrm{l})$ at the time of enrollment.

Patients were excluded if they had a history of dose intensification within the last 12 months of biological therapy, history of relapse after ceasing biological therapy in the past, history of more than one intestinal resection for $\mathrm{CD}$, intestinal fibrotic stenosis (defined as narrowing of the intestinal lumen by $<10 \mathrm{~mm}$ on endoscopy, computed tomography or magnetic resonance imaging enterography, or transabdominal small bowel ultrasound), regardless of whether it was symptomatic or asymptomatic, history of rheumatoid arthritis, ankylosing spondylitis or psoriasis, or any other severe disease whose worsening on relapse of IBD might pose a risk to the patient. Pregnant patients were also excluded.

\section{The Clinical cohort}

These IBD patients stopped biological therapy after at least 12 months of treatment for other subjective or medical reasons and at the time of stopping were in clinical remission. The patients were enrolled in the Clinical cohort during the same time period as the Ultradeep cohort patients. The reasons for discontinuing anti-TNF $\alpha$ therapy included the patient's own decision to stop biological therapy, severe adverse events, and/or severe infection.

Assessment of clinical activity and definition of clinical remission

Clinical activity was assessed by Harvey-Bradshaw Index (HBI) for patients with Crohn's disease and with partial Mayo score for patients with ulcerative colitis $(15,16)$. CD patients were deemed to be in clinical remission if they had a HBI score of $<5$ with no active fistula, while UC patients were considered to be in clinical remission when their pMayo score was $<2$.

Mucosal healing was defined as endoscopic Mayo subscore 0 for UC patients and lack of ulcers, erosions, and marked hyperemia on ileocolonoscopy for CD patients. Pseudopolyps that lacked signs of inflammatory activity did not exclude the definition of mucosal healing.

\section{Follow-up and definition of clinical relapse}

After ceasing anti-TNF $\alpha$ therapy, all baseline medication, including immunosuppressives, were continued until the last followup or relapse. All patients were followed-up at 3-month intervals for the first 12 months and then every 6 months until April 2015 . At each visit, blood was taken to determine the complete blood counts and serum CRP levels, and stool was collected for the measurement of FC levels. Moreover, the clinical activity of CD and UC patients was determined by using the HBI and pMayo scores, respectively $(15,16)$.

CD patients were deemed to be in clinical relapse if their HBI scores increased by $>2$ points and became $>4$ points or a fistula opened. UC patients were considered to be in clinical relapse if their pMayo score increased by $>2$ points. If patients who had a clinical relapse lacked contraindications and the patient consented, 
Tab. 1. Baseline clinical characteristics of the cohort.

\begin{tabular}{lcc}
\hline \multirow{2}{*}{ Baseline characteristics } & \multicolumn{2}{c}{ Cohort } \\
\cline { 2 - 3 } & $\begin{array}{c}\text { Clinical remission } \\
(\mathrm{n}=11)\end{array}$ & $\begin{array}{c}\text { Ultradeep remission } \\
(\mathrm{n}=11)\end{array}$ \\
\hline CD/UC & $8 / 3$ & $9 / 2$ \\
\hline Females (\%) & $7(64 \%)$ & $5(46 \%)$ \\
\hline Median age & 36.4 & 35.8 \\
(range), yrs & $(27.2-74.7)$ & $(24.1-73.3)$ \\
\hline Median disease & 8.5 & 5.8 \\
duration (range), yrs & $(2.3-19.7)$ & $(3.1-17.2)$ \\
\hline Location* & $L 1: 3$ & $L 1: 2$ \\
& $L 2: 2$ & $L 2: 3$ \\
& $L 3: 2$ & $L 3: 3$ \\
& $L 4: 1$ & $L 4: 1$ \\
\hline Behavior - CD* & $E 3: 3$ & $E 2: 2$ \\
\hline Smoking (\%) & $B 1: 2$ & $B 1: 3$ \\
\hline Major IBD surgery & $B 2: 3$ & $B 2: 2$ \\
\hline Infliximab/adalimumab & $B 3: 3$ & $B 3: 4$ \\
\hline Median duration of anti- & $2(18 \%)$ & 0 \\
TNF $\alpha$ therapy (range), yrs & $4(36 \%)$ & $3(27 \%)$ \\
\hline AZA (\%) & $9 / 2$ & $9 / 2$ \\
\hline 5-ASA (\%) & 3.4 & 4.0 \\
\hline * According to the Montreal classification (14). CD - Crohn's disease; UC - ul- & $(1.5-7.2)$ \\
cerative colitis; IBD - inflammatory bowel disease; AZA- azathioprin; anti-TNF \\
- anti-tumor necrosis factor alpha; 5-ASA-5-aminosalicylic acid \\
\hline
\end{tabular}

reinduction therapy with anti-TNF $\alpha$ was initiated. Whether the patient again achieved clinical remission (HBI score of $<5$ with no active fistula for CD patients and pMayo score of $<2$ for UC patients) was assessed 14 weeks after starting the reinduction therapy.

The disease-related quality of life was measured at each visit by using the short inflammatory bowel disease questionnaire (sIBDQ) (17). In our center, an sIBDQ score of $\geq 50$ (maximum 70) is considered to indicate normal for both UC and CD patients (18).

\section{Primary and secondary study endpoints}

The primary endpoint was the cumulative relapse rate 1 year after ceasing anti-TNF $\alpha$ therapy in each group. Secondary endpoints were as follows: 1) cumulative relapse rate 2 years after ceasing biological therapy in each group, 2) difference between the two groups in terms of median sIBDQ scores and FC levels 1 year after treatment discontinuation in non-relapsing patients, 3) predictors of relapse, and 4) response to reinduction therapy with anti-TNF $\alpha$ by patients who relapsed during the follow-up.

\section{Assessment of FC levels}

The stool samples were collected the day before or during the day of the study visit. Patients were advised to keep the samples in a closed container in a home refrigerator. On the day of the study visit, the samples were transported to the hospital clinical biochemistry laboratory at room temperature. FC levels were measured by using a quantitative enzyme-linked immunosorbent assay (CalproLab, Oslo, Norway). FC levels below $50 \mathrm{ng} / \mathrm{g}$ of stool were considered normal.

\section{Statistical analyses}

All assessed variables were defined prior to data collection. Statistical tests were performed by using SPSS 19.0 (IBM SPSS Inc., Chicago, Illinois, USA). All p-values are two-sided and pvalues $<0.05$ were considered to indicate statistical significance. Descriptive statistics are presented as percentages (categorical variables) and median with range (continuous variables). Time until relapse was analyzed by using the Kaplan-Meier method and Cox regression analysis was used to compare the survival curves. The change in SIBDQ scores and calprotectin levels 12 months after ceasing biological treatment relative to baseline was analyzed by using the Wilcoxon test. Univariate analysis of the association of baseline characteristics with relapse at 1 year was assessed by using Fisher's exact test (categorical variables) or the Mann-Whitney U-test (continuous variables). Variables were also assessed for their association with relapse by using univariate Cox proportional hazard regression analysis.

\section{Ethical considerations}

The study protocol was reviewed and approved by the Ethics Committee of University Hospital Bratislava. Patients in the Ultradeep cohort gave written informed consent to participate in the study. All subjects gave written approval regarding the use of their clinical and laboratory data in the study analyses.

\section{Results}

\section{Study population}

The study population consisted of 22 IBD patients. The U1tradeep cohort had 11 patients (eight with $\mathrm{CD}$, three with UC). The Clinical cohort also had 11 patients (nine with $\mathrm{CD}$, two with UC). Table 1 indicates the clinical and demographic characteristics of the two cohorts at the time they ceased anti-TNF $\alpha$ therapy. The two groups did not differ significantly in terms of the baseline characteristics except that the Ultradeep cohort was more likely to be on concomitant immunosuppressive therapy ( $64 \%$ vs $36 \%$, p = 0.20 ). The majority of patients in the two cohorts (both $9 / 11$ ) were receiving infliximab at the time of stopping. Both patients in the Clinical cohort who were receiving adalimumab had CD. The two patients in the Ultradeep cohort on adalimumab had CD and UC.

Seven of the Clinical cohort patients stopped anti-TNF $\alpha$ therapy at their own behest for various reasons (e.g. long-term travel, fear of side effects). The remaining four patients stopped because of the development of severe skin psoriatiform lesions $(n=1)$, retinal melanoma $(n=1)$, posttraumatic leg abscess with phlegmona $(n=1)$, and severe joint pain $(n=1)$. In eight Clinical cohort patients, the FC status before stopping anti-TNF $\alpha$ therapy was known: one patient was normal ( $\mathrm{FC}<50 \mathrm{ng} / \mathrm{g}$ ), four patients had levels between 50 and $300 \mathrm{ng} / \mathrm{g}$, and three patients had levels exceeding $300 \mathrm{ng} / \mathrm{g}$. The patient with normal FC levels had short bowel syndrome after undergoing multiple intestinal resections.

\section{Cumulative relapse rate}

The median follow-up periods for the Ultradeep and Clinical cohorts were 497 (range, 230-1249) days and 591 (range, 130- 


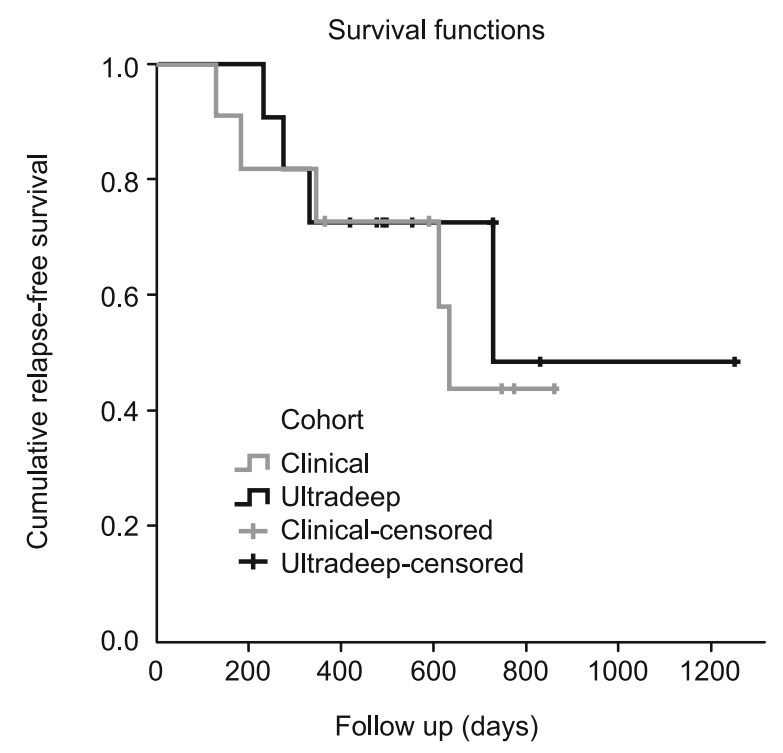

Fig. 1. Cumulative relapse rate after discontinuation of anti-tumor necrosis factor $\alpha$ therapy.

861) days. In both Ultradeep and Clinical cohorts, two (18\%) and three $(27 \%)$ patients relapsed within 6 and 12 months after ceasing anti-TNF $\alpha$ therapy $(p=0.85)$. All of the six patients who relapsed within 1 year were CD patients who stopped infliximab therapy. Within 2 years after ceasing anti-TNF $\alpha$ therapy, another Ultradeep cohort patient (CD) and two Clinical cohort patients (one with CD, one with UC) had relapsed. All had ceased infliximab therapy.

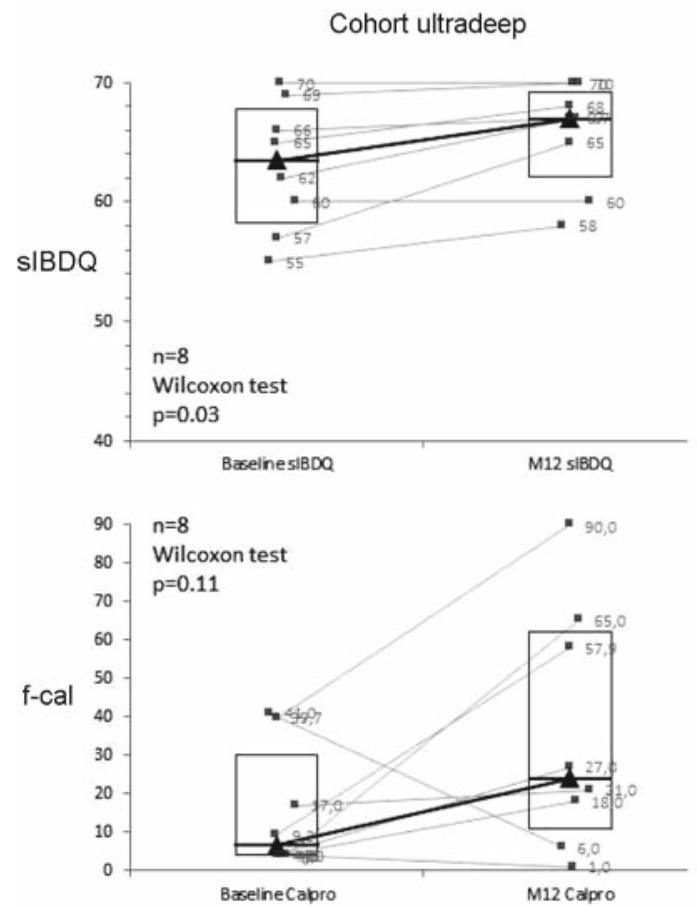

Thus, by the end of the second year after ceasing anti-TNF $\alpha$ therapy, four of the Ultradeep patients had relapsed, three had been still in sustained clinical remission, and one had been lost to followup. In the Clinical cohort, by the end of the second year, five patients had relapsed, three had been in sustained clinical remission, and one had been lost to follow-up. Figure 1 shows the Kaplan-Meier survival curves of the cumulative relapse rates of the two groups after anti-TNF $\alpha$ therapy cessation over the 2-year follow-up period. For the Ultradeep and Clinical cohorts, the median time to relapse was 635 and 730 days, respectively. The two groups did not differ significantly in terms of cumulative relapse rates (log-rank test, $p=0.73$ ).

Change relative to baseline in $I B D Q$ scores and $F C$ levels one year after ceasing anti-TNF $\alpha$ therapy

The patients in the cohorts who were in sustained clinical remission 1 year after ceasing biological therapy (both $n=8$ ) were then assessed for changes relative to baseline in terms of SIBDQ scores and FC levels. Figure 2 shows that the eight Ultradeep patients exhibited a small but significant increase in SIBDQ scores: the median scores at baseline and 1 year were 63.5 and 67, respectively (Wilcoxon test $\mathrm{p}=0.03$ ). The eight Clinical patients also exhibited a small increase in SIBDQ scores but this did not achieve statistical significance. The FC levels rose in the Ultradeep patients and fell in the Clinical patients, but these changes also did not achieve statistical significance.

Factors associated with relapse

Univariate analyses were performed to identify the risk factors that associated with relapse within the first year after anti-TNF $\alpha$

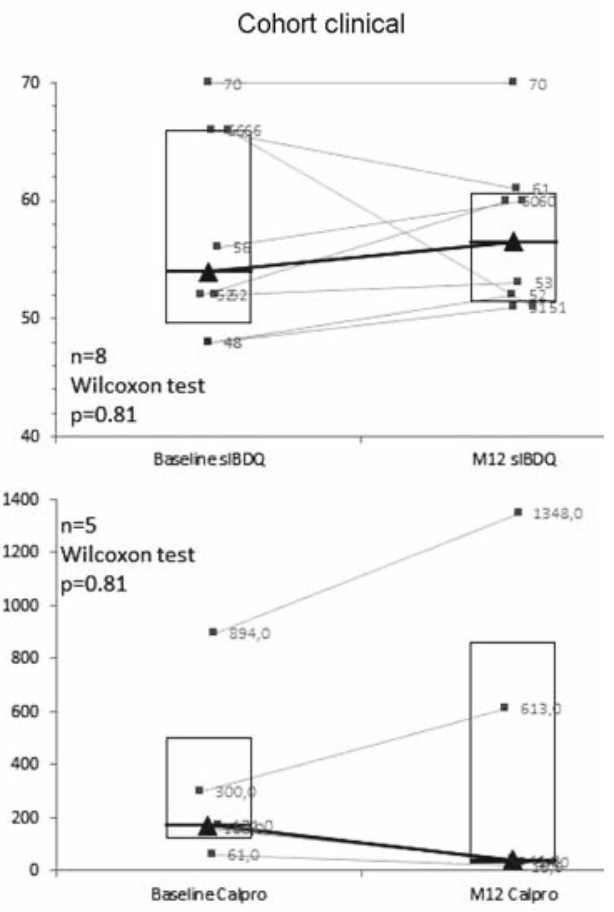

Fig. 2. Change relative to baseline in short inflammatory bowel disease questionnaire scores and fecal calprotectin levels 12 months after inflammatory bowel disease patients in sustained clinical remission ceased anti-tumor necrosis factor $\alpha$ therapy. 


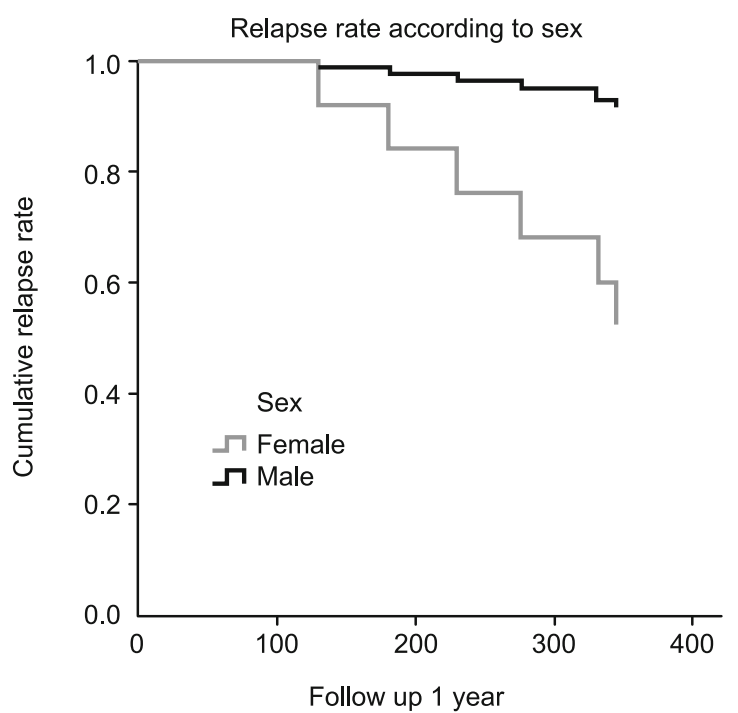

Fig. 3. Cumulative relapse rate after discontinuation of anti-tumor necrosis factor $\alpha$ therapy in males and females.

therapy cessation in the whole cohort $(n=22)$ (Tab. 2). The only significant risk factor of relapse was male sex: five of the ten $(50$ $\%)$ males relapsed, whereas only one of the twelve ( $8.3 \%)$ females relapsed $(\mathrm{p}=0.03)$. CD patients also tended to relapse more frequently than UC patients $(6 / 17,35 \%$ vs $0 / 5)$, but this difference did not achieve statistical significance $(\mathrm{p}=0.12)$. Cox regression analysis of the association of sex with relapse showed a tendency for males to experience relapse more frequently than females, but this difference did not achieve statistical significance (Hazard Ratio (HR): 6.9, 95\% confidence intervals (CIs): $0.8-58.9, \mathrm{p}$ $=0.08)$ (Fig. 3).

\section{Efficacy and safety of retreatment with anti-TNFa in patients who relapsed}

In total, nine IBD patients (eight with $\mathrm{CD}$, one with $\mathrm{UC}$ ) relapsed in the follow-up period. Seven patients were re-treated with infliximab and all achieved clinical remission by week 14 after starting retreatment. There were no infusion reactions or other adverse events on the reintroduction of infliximab. The two patients who had relapsed but were not re-treated with anti-TNF $\alpha$ were CD patients in the Clinical group; both worked abroad. During the remaining follow-up period, one of these patients was admitted to hospital and treated with corticosteroids, and the re-opened fistula of the second patient was treated symptomatically.

\section{Discussion}

This prospective single-center open-label study assessed the relapse rates of 22 IBD patients who stopped anti-TNF $\alpha$ therapy, either on physician recommendations because they were in deep remission with normal FC levels $(<50 \mu \mathrm{g} / \mathrm{g})$ (11 Ultradeep patients) or because they were in clinical but not deep remission and stopped for other reasons (11 Clinical patients). Within the first year after stopping, $27 \%$ of the patients in both cohorts relapsed.
By the second year after stopping, $57 \%$ of the Ultradeep cohort and $62 \%$ of the Clinical cohort had relapsed.

\section{Relapse rates in other studies}

At present, there are no published reports of randomized controlled trials examining the relapse rate after anti-TNFacessation in IBD patients: all published studies on this matter are observational. These include three retrospective and six prospective studies (11-13,19-24). All nine studies included rather few patients (14 to 121 ), all but two included only infliximab stoppers, and only three studies included UC patients.

The retrospective studies had longer follow-up periods than the prospective studies and relapses were defined as the introduction of new IBD treatment, abdominal surgery, or hospital admission. The most recent retrospective study was from Leuven: the cohort consisted of $100 \mathrm{CD}$ patients who were in clinical remission and stopped biological therapy. The 1- and 2-year cumulative relapse rates were very low: $4 \%$ and $7 \%$, respectively (23). Even after a median follow-up period of 9.7 years, $52 \%$ of the CD patients in this cohort were still in clinical remission after infliximab discontinuation. By contrast, the retrospective study of Steenholdt et al on $53 \mathrm{CD}$ patients and $28 \mathrm{UC}$ patients who stopped infliximab while in corticosteroid-free clinical remission reported that within 2 years of the ceasing treatment, approximately $50 \%$ and $30 \%$ of the CD and UC patients relapsed, respectively (20).

The prospective studies evaluated the relapse rates on the basis of clinical activity indices and had shorter follow-up periods than the retrospective studies. Louis et al performed a prospective multicenter open-label trial (STORI) with $115 \mathrm{CD}$ patients who stopped infliximab after having been in steroid-free clinical remission for at least 6 months (13). They continued their therapy with azathioprine. The median follow-up period was 28 months and the 1- and 2 -year relapse rates were $44 \%$ and $52 \%$, respectively. Similarly, Farkas et al. performed a prospective multicenter observational study with $121 \mathrm{CD}$ patients who were followed up for 12 months after stopping infliximab (87 patients) and adalimumab (34\%) therapy. The 6-month cumulative relapse rate was $45 \%$. The only prospective observational study that enrolled UC patients $(n=51)$ reported a 1-year relapse rate of $35 \%$ (22). Finally, there was a recent report of an open-label multicenter trial in Finland with 51 IBD patients who were (as in our study) in clinical, endoscopic, and FC-negative $(<100 \mu \mathrm{g} / \mathrm{g})$ remission and stopped anti-TNF $\alpha$ therapy (either infliximab or adalimumab). The median followup period was 13 months. Similar to our study, the 1-year relapse rate was $33 \%$ and no difference between $\mathrm{CD}$ and UC patients in terms of relapse rates was observed.

Thus, the relapse rates reported in our study are generally similar to those reported by other studies.

\section{Risk factors of relapse}

The only significant risk factor for relapse in our cohort was male sex: $50 \%$ of the males relapsed within 1 year, whereas only $11 \%$ of the females relapsed. This is consistent with observations from the STORI study: the HR estimate for male sex was 3.7 (13). Based on univariate analyses, the STORI trial also found with uni- 
Tab. 2. Identification of factors that associate with relapse within one year.

\begin{tabular}{|c|c|c|c|c|}
\hline Factor at baseline & & $\begin{array}{c}\text { Relapse within } 1 \text { year } \\
(\mathrm{n}=6)\end{array}$ & $\begin{array}{c}\text { No relapse within } 1 \text { year } \\
(\mathrm{n}=16)\end{array}$ & $\mathrm{p}$ value** \\
\hline Diagnosis & $\begin{array}{l}\mathrm{CD} \\
\mathrm{UC}\end{array}$ & $\begin{array}{c}6(35 \%) \\
0 \\
\end{array}$ & $\begin{array}{l}11(65 \%) \\
5(100 \%) \\
\end{array}$ & 0.12 \\
\hline$\overline{\operatorname{Sex}}$ & $\begin{array}{c}\text { Male } \\
\text { Female }\end{array}$ & $\begin{array}{l}5(50 \%) \\
1(8 \%) \\
\end{array}$ & $\begin{array}{c}5(50 \%) \\
11(92 \%) \\
\end{array}$ & 0.03 \\
\hline Median age, yrs & & 38.2 & 31.0 & 0.16 \\
\hline Median disease duration, yrs & & 7.1 & 8.9 & 0.94 \\
\hline Location - CD* & $\begin{array}{l}\mathrm{L} 1 \\
\mathrm{~L} 2 \\
\mathrm{~L} 3 \\
\mathrm{~L} 4 \\
\end{array}$ & $\begin{array}{l}1 \\
1 \\
3 \\
1 \\
\end{array}$ & $\begin{array}{l}4 \\
4 \\
2 \\
1 \\
\end{array}$ & 0.37 \\
\hline Behavior - CD* & $\begin{array}{l}\text { B1 } \\
\text { B2 } \\
\text { B3 }\end{array}$ & $\begin{array}{l}1 \\
2 \\
3 \\
\end{array}$ & $\begin{array}{l}4 \\
3 \\
4 \\
\end{array}$ & 0.35 \\
\hline Smoking & $\begin{array}{l}\text { Yes } \\
\text { No }\end{array}$ & $\begin{array}{l}1(50 \%) \\
5(25 \%) \\
\end{array}$ & $\begin{array}{c}1(50 \%) \\
15(20 \%) \\
\end{array}$ & 0.45 \\
\hline IBD surgery & $\begin{array}{l}\text { Yes } \\
\text { No }\end{array}$ & $\begin{array}{l}2(29 \%) \\
4(27 \%) \\
\end{array}$ & $\begin{array}{c}5(71 \%) \\
11(73 \%)\end{array}$ & 0.92 \\
\hline Type of anti-TNF $\alpha$ therapy & $\begin{array}{l}\text { IFX } \\
\text { ADA } \\
\end{array}$ & $\begin{array}{c}6(33 \%) \\
0 \\
\end{array}$ & $\begin{array}{l}12(67 \%) \\
4(100 \%) \\
\end{array}$ & 0.18 \\
\hline Median duration of anti-TNF $\alpha$ therapy, yrs & & 3.7 & 4.2 & 0.24 \\
\hline$\overline{\mathrm{AZA}}$ & $\begin{array}{l}\text { Yes } \\
\text { No }\end{array}$ & $\begin{array}{l}3(27 \%) \\
3(27 \%) \\
\end{array}$ & $\begin{array}{l}8(73 \%) \\
8(73 \%) \\
\end{array}$ & 1.0 \\
\hline Median HBI score & & 1 & 0.5 & 0.59 \\
\hline Median sIBDQ score & & 68 & 61 & 0.15 \\
\hline Median CRP level, mg/L & & 1 & 1 & 1 \\
\hline Median $\mathrm{Hb}$ level, g/L & & 148 & 142 & 0.29 \\
\hline Median fecal calprotectin level, $\mu \mathrm{g} / \mathrm{g}$ & & 43 & 39 & 0.86 \\
\hline
\end{tabular}

variate analyses that the following factors associated significantly with an increased relapse rate: corticosteroid use 6-12 months before baseline (HR: 3.5, 95\% CIs: 1.1-10.7), no previous surgical resection (HR: $4.0,95 \%$ CIs: $1.4-11.4$ ), leukocyte count $>6 \times 10^{9}$ (HR: 2.4, 95\% CIs: 1.2-4.7), Crohn disease endoscopic severity score (CDEIS) $>0$ (HR: $2.3,95 \%$ CIs: 1.1-4.9), hemoglobin level $\leq 145$ g/l (HR: $6.0,95 \%$ CIs: $2.2-16.5$ ), high sensitive C-reactive protein level $\geq 5 \mathrm{mg} / \mathrm{L}$ (HR: $3.2,95 \%$ CIs: 1.6-6.4), infliximab trough level $2 \mathrm{mg} / \mathrm{L}$ (HR: 2.5, 95\% CIs: 1.1-5.4), and FC levels $\geq 300 \mu \mathrm{g} / \mathrm{g}$ (HR: 2.5, 95\% CIs: 1.1-5.8) (13). Patients with no more than two of these risk factors (approximately $29 \%$ of the study population) had a $15 \%$ risk of relapse within 1 year. Other studies reported that the following factors associate with the risk of relapse after infliximab discontinuation: longer disease duration at the time of first infliximab infusion (CD patients), previous biological therapy (both CD and UC patients), previous dose intensification (CD patients), and age at the time of diagnosis of less than 25 years (CD patients) (20-23) .

\section{Effectiveness of retreatment}

In our study, seven patients were re-treated with infliximab. All achieved clinical remission within 14 weeks. This is consistent with other studies, which reported $93-96 \%$ remission rates in $\mathrm{CD}$ patients and a $71 \%$ remission rate in UC patients within 14 weeks of infliximab retreatment $(13,20,24)$.

\section{Study limitations}

Our study has several limitations. Firstly, the cohort size was small $(\mathrm{n}=22)$, which limited the statistical power of the study. As a result, only substantial differences could be detected. This limitation means that our study is essentially an observational study, despite its clinical trial-like design. Secondly, the patients were heterogeneous: both $\mathrm{CD}$ and UC patients, and patients treated with different anti-TNF $\alpha$ agents (infliximab and adalimumab) were included. Although our exploratory statistical analyses suggested that $\mathrm{CD}$ and UC patients, and patients being treated with infliximab and adalimumab do not differ significantly in terms of relapse rates, it remains possible that the heterogeneity of our cohort is a confounding factor in our analyses.

\section{Clinical implications}

Our study showed that even highly selected IBD patients, who lack clinical, endoscopic or laboratory signs of inflammatory activity and have not had major structural damage to the bowel have a relatively high relapse rate $(27 \%)$ within 1 year after ceasing anti$\mathrm{TNF} \alpha$ therapy. However, the reintroduction of anti-TNF $\alpha$ therapy 
in these patients seemed to be effective and safe. The risks and benefits of stopping anti-TNF $\alpha$ therapy must be discussed with each patient on individual basis. Our study and those of others do not support the general recommendation to stop anti-TNF $\alpha$ therapy even when deep remission is achieved and the FC levels are normal. Further studies that identify IBD populations that have a low risk of relapse after stopping anti-TNF $\alpha$ therapy are warranted.

\section{References}

1. Rutgeerts P, Sandborn WJ, Feagan BG, Reinisch W, Olson A, Johanns $\mathbf{J}$ et al. Infliximab for induction and maintenance therapy for ulcerative colitis. N Engl J Med 2005; 353 (23): 2462-2476.

2. Hanauer SB, Feagan BG, Lichtenstein GR, Mayer LF, Schreiber S, Colombel JF et al. Maintenance infliximab for crohn's disease: The ACCENT I randomised trial. The Lancet 2002; 359 (9317): 1541-1549.

3. Colombel JF, Sandborn WJ, Rutgeerts P, Enns R, Hanauer SB, Panaccione $\mathbf{R}$ et al. Adalimumab for maintenance of clinical response and remission in patients with crohn's disease: The CHARM trial. Gastroenterology 2007; 132 (1): 52-65.

4. Hazlewood GS, Rezaie A, Borman M, Panaccione R, Ghosh S, Seow CH et al. Comparative effectiveness of immunosuppressant and biologics for inducing and maintaining remission in crohn's disease: A network meta-analysis. Gastroenterology 2014; 0 (0).

5. Lv R, Qiao W, Wu Z, Wang Y, Dai S, Liu Q, Zheng X. Tumor necrosis factor alpha blocking agents as treatment for ulcerative colitis intolerant or refractory to conventional medical therapy: A meta-analysis. PLoS On e 2014; 9(1): e86692.

6. Colombel JF, Loftus EVJ, Tremaine WJ, Egan LJ, Harmsen WS, Schleck CD et al. The safety profile of infliximab in patients with crohn's disease: The mayo clinic experience in 500 patients. Gastroenterology 2004; 126 (1): 19-31.

7. Lichtenstein GR, Feagan BG, Cohen RD, Salzberg BA, Diamond RH, Chen DM et al. Serious infections and mortality in association with therapies for crohn's disease: TREAT registry. Clin Gastroenterol Hepatol 2006; 4 (5): 621-630.

8. Peyrin-Biroulet L, Deltenre P, de Suray N, Branche J, Sandborn WJ, Colombel JF. Efficacy and safety of tumor necrosis factor antagonists in Crohn's disease: Meta-analysis of placebo-controlled trials. Clin Gastroenterol Hepatol 2008; 6 (6): 644-653.

9. Burisch J, Vardi H, Pedersen N, Brinar M, Cukovic-Cavka S, Kaimakliotis I et al. Costs and resource utilization for diagnosis and treatment during the initial year in a european inflammatory bowel disease inception cohort: An ecco-epicom study. Inflamm Bowel Dis 2015; 21 (1): 121-131.

10. Pittet V, Froehlich F, Maillard MH, Mottet C, Gonvers JJ, Felley C et al. When do we dare to stop biological or immunomodulatory therapy for crohn's disease? Results of a multidisciplinary european expert panel. J Crohns Colitis 2013; 7 (10): 820-826.

11. Domènech E, Hinojosa J, Nos P, Garcia-Planella E, Cabré E, Bernal I, Gassull MA. Clinical evolution of luminal and perianal crohn's disease after inducing remission with infliximab: How long should patients be treated? Aliment Pharmacol Ther 2005; 22 (11-12): 1107-1113.
12. Waugh AW, Garg S, Matic K, Gramlich L, Wong C, Sadowski DC et al. Maintenance of clinical benefit in crohn's disease patients after discontinuation of infliximab: Long-term follow-up of a single centre cohort. Aliment Pharmacol Ther 2010; 32 (9): 1129-1134.

13. Louis E, Mary JY, Vernier-Massouille G, Grimaud JC, Bouhnik Y, Laharie D et al. Maintenance of remission among patients with crohn's disease on antimetabolite therapy after infliximab therapy is stopped. Gastroenterology 2012; 142 (1): 63-70.

14. Silverberg MS, Satsangi J, Ahmad T, Arnott ID, Bernstein CN, Brant SR et al. Toward an integrated clinical, molecular and serological classification of inflammatory bowel disease: Report of a working party of the 2005 montreal world congress of gastroenterology. Can J Gastroenterol 2005; 19 Suppl A: 5A-36A.

15. Sutherland LR, Martin F, Greer S, Robinson M, Greenberger N, Saibil F et al. 5-Aminosalicylic acid enema in the treatment of distal ulcerative colitis, proctosigmoiditis, and proctitis. Gastroenterology 1987; 92 (6): 1894-1898.

16. Harvey RF, Bradshaw JM. A simple index of crohn's-disease activity. Lancet 1980; 1(8167): 514, Pubmed ID: http://www.ncbi.nlm.nih. gov/pubmed/6102236

17. Irvine EJ, Zhou Q, Thompson AK. The short inflammatory bowel disease questionnaire: A quality of life instrument for community physicians managing inflammatory bowel disease. American Journal of Gastroenterology 1996; 91 (8).

18. Hlavaty T, Persoons P, Vermeire S, Ferrante M, Pierik M, Van Assche G, Rutgeerts P. Evaluation of short-term responsiveness and cutoff values of inflammatory bowel disease questionnaire in crohn's disease. Inflamm Bowel Dis 2006; 12 (3): 199-204.

19. Lu C, Waugh A, Bailey RJ, Cherry R, Dieleman LA, Gramlich L et al. Crohn's disease genotypes of patients in remission vs relapses after infliximab discontinuation. World J Gastroenterol 2012; 18 (36): 5058-5064.

20. Steenholdt C, Molazahi A, Ainsworth MA, Brynskov J, Østergaard Thomsen O, Seidelin JB. Outcome after discontinuation of infliximab in patients with inflammatory bowel disease in clinical remission: An observational danish single center study. Scand J Gastroenterol 2012; 47 (5): 518-527.

21. Molnár T, Lakatos PL, Farkas K, Nagy F, Szepes Z, Miheller P et al. Predictors of relapse in patients with crohn's disease in remission after 1 year of biological therapy. Aliment Pharmacol Ther 2013; 37 (2): 225-233.

22. Farkas K, Lakatos PL, Nagy F, Szepes Z, Miheller P, Papp M et al. Predictors of relapse in patients with ulcerative colitis in remission after one-year of infliximab therapy. Scand J Gastroenterol 2013; 48 (12): 1394-1398.

23. Papamichael K, Casteele NV, Gils A, Tops S, Hauenstein S, Singh S et al. Long-Term outcome of patients with crohn's disease who discontinued infliximab therapy upon clinical remission. Clin Gastroenterol Hepatol 2014.

24. Molander P, Färkkilä M, Salminen K, Kemppainen H, Blomster T, Koskela R et al. Outcome after discontinuation of tnf $\alpha$-blocking therapy in patients with inflammatory bowel disease in deep remission. Inflamm Bowel Dis 2014; 20 (6): 1021-1028. 